\section{Data "Repair-Kit" Developed for Quantum Computing}

J. Chiaverini and colleagues at the National Institute of Standards and Technology have developed and demonstrated a method for automatically correcting data-handling errors in quantum computation. Described in the December 2, 2004, issue of Nature (p. 602; doi:10.1038/ nature03074), a set of three atomic qubits are used to detect and correct data-handling errors in one of the qubits.

"The basic concept is a familiar one: If someone doesn't understand what you say, you repeat it several times, and eventually they'll get it," said physicist D. Leibfried, who developed the approach and helped to demonstrate its feasibility.

In this method, three beryllium ions are held in a micrometer-sized electromagnetic trap, where their quantum states are manipulated with ultraviolet laser beams and their physical movement is controlled with electrodes. One ion is the primary qubit containing the data essential to a computation; the other two are "helper" qubits.

An ion's quantum state includes properties such as the orientation of its spin. The digital values 1 and 0 are represented by the direction of the spin. "Spin-up," corresponding to 0 , has a greater energy than "spin-down," corresponding to 1 .

Because qubits can exist in both states simultaneously, a condition called "quantum superposition," they can represent both 0 and 1 at the same time. On a sphere representing all possible spin states, a superposition can be visualized as a tilt of the north-south axis, at an angle that is dependent on whether there is a greater probability of the 0 state or the 1 state. When a qubit is measured by probing it with a laser, the state collapses to 0 or 1 , with a rate of occurrence that depends on the angle of the ion's tilt (i.e., the probability of its being 1 or 0 ). For example, when the ion's axis points directly to the side (i.e., the axis is horizontal), the qubit will have an equal probability of collapsing to spin-up or to spin-down. For each single experiment, it will collapse to one of the two states; but if the experiment is repeated 100 times, the qubit will, on average, collapse to spin-down 50 times, and to spin-up 50 times.

The researchers prepared the primary qubit in a superposition of spin-up and spin-down. They then encoded the data in this qubit into the set of three qubits by putting the three ions through a series of quantum logic gates. This has the effect of correlating the quantum properties of all three qubits so that an action on one pro- duces a predictable outcome on the others. One type of logic gate places the two extra ions in superposition states. Another type of logic gate has the effect of entangling the three ions, so their quantum states are related to each other in a particular choreography. Overall, these processing steps place the entangled system in a superposition of several possible quantum spin states of the three atoms.

The researchers used lasers to introduce errors - spin flips, or reversals-into all of the qubits simultaneously, as might occur in a real computer due to environmental disturbances, at various rates. The exact nature of the errors occurring in a single run of the experiment depended partly on the length of the laser pulse hitting the ions and partly on the unusual features of quantum mechanics, which produces results that can be predicted only in terms of probabilities.

The quantum logic operations were then performed in reverse order to "decode" the data in the entangled system. The process was designed so that the qubit state needed for the computation was transferred back to the primary qubit, and the error information was contained in the two extra qubits. These two were measured by hitting them with a different laser pulse that either made them fluoresce if they were spin-down or remain dark if they were spin-up. This procedure forced their states to collapse to one of four possible outcomes (spin-up/spin-up, spin-down/spin-up, spin-up/spin-down, or spin-down/spin-down), depending on the error that occurred.

At the time of measurement, the primary qubit was disentangled from the helper qubits and left in either its original superposition state or one of two slightly altered versions of that state, rotated around a particular axis. The final values of the two helper qubits heralded which of these cases occurred, so that corrections could be made if needed. For instance, the routine was designed so that if both extra ions were spin-down, then the spin of the primary qubit was flipped around a particular axis and could be corrected with a precisely timed laser pulse to undo that flip. The two extra qubits could then be reset and used again to detect and correct future errors. The researchers noted that their quantum error correction code "works perfectly only when at most one of the qubits undergoes a spin-flip error." They

FOR MORE RESEARCH NEWS ON MATERIALS SCIENCE. access the Materials Research Society Web site: www.mrs.org/connections also noted that some infidelity was introduced by application of their quantum error correction code, but that that was due largely to problems with the fidelity of the coding and decoding gates, and that "all operations must be improved to achieve fault-tolerance."

\section{Quantum Dots Fabricated in Silica Aerogel by Photolithographic Approach}

M.F. Bertino and colleagues from the University of Missouri-Rolla, S. Guha from the University of Missouri-Columbia, and N. Leventis from the NASA Glenn Research Center have created quantum dots embedded in a silica aerogel with the use of a photolithographic technique. As reported in the December 13, 2004, issue of Applied Physics Letters (p. 6007; doi: $10.1063 / 1.1836000)$, the researchers used localized laser heating to initiate a chemical reaction within a cooled silica hydrogel into which cooled solutions of chemical precursors had been mixed. The silica hydrogel can be dried later in supercritical $\mathrm{CO}_{2}$ to form an aerogel.

The hydrogels were cooled to $5^{\circ} \mathrm{C}$ and then bathed in precooled aqueous solutions of $\mathrm{Cd}\left(\mathrm{NO}_{3}\right)_{2}$ and $\mathrm{NH}_{4} \mathrm{OH}$. After a short bathing period, half of the bathing solution was replaced by a cooled thiourea solution. When the precursor materials are mixed at room temperature they react to form the semiconductor cadmium sulfide. Hydrogels loaded with the precursor solutions turned yellow (indicating the formation of $\mathrm{CdS}$ ) within $1 \mathrm{~h}$ at room temperature. However, the reaction is strongly reduced at low temperature. After cooling the mixture to halt the chemical reaction, Bertino and his team placed the silica gel in front of the infrared laser. By sharply focusing to reduce the laser's beam width to a few microns, they heated small, isolated sections of the silica gel. The chemical reaction caused by the heat formed the semiconductor particles-or quantum dots—only in the heated regions.

"Our cadmium sulfide quantum dots not only absorb light but they also emit light," Bertino said. "By tuning the size of the particles, you can change the emission range." Such properties could also be of interest to scientists who hope to develop quantum computers, quantum-dot lasers, or molecular-scale integrated circuits, Bertino said. 